Jetzt aber schnell

\title{
Nominierungsfrist endet: Pflegemanagement-Award 2017
}

— Noch bis zum 30. September 2016 können Führungskräfte in der Pflege für den Pflegemanagement-Award 2017 nominiert werden. Und diesmal gleich in zwei Kategorien. Gesucht werden der/die ,Nachwuchs-

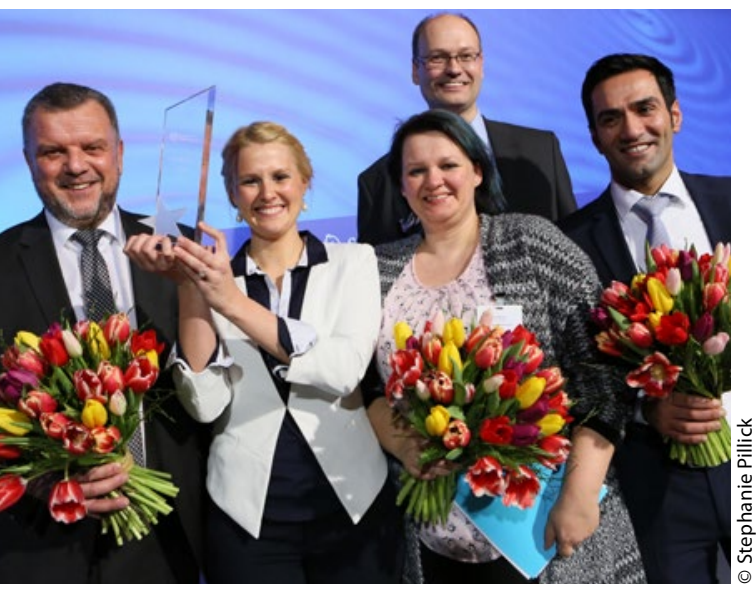

Sarah Behling, Doreen Hoffmann und Mustafa Atas waren die Sieger 2016

\begin{abstract}
Pflegemanager/in' und der/die ,Pflegemanager/in des Jahres. Beide Auszeichnungen werden vom Bundesverband Pflegemanagement auf dem von Springer ausgerichteten "Kongress Pflege 2017" verliehen. Hauptsponsor der Preisverleihung 2017 ist bereits zum fünften Mal in Folge ZeQ - eine der führenden Unternehmensberatungen für Krankenhäuser, Psychiatrien und Rehakliniken.

In beiden Kategorien sorgt eine hochkarätige Jury bestehend aus Vertretern aus Politik, Wirtschaft, Wissenschaft sowie dem Verbands- und Verlagswesen für eine professionelle Bewertung der Bewerber. In der Kategorie ,Nachwuchs-Pflegemanager/in des Jahres' werden erneut Nachwuchsführungskräfte gesucht, die sich mit Engagement, Kreativität sowie sozialer und fachlicher Kompetenz für die Stärkung der Position ihrer Profession einsetzen. Und dabei die für ihren Beruf so wichtige Empathie nicht aus den Augen verlieren.
\end{abstract}

In der Kategorie ,Pflegemanager/in des Jahres' werden Manager der Top-Ebene gesucht, die die pflegerische Gesamtverantwortung für eine Einrichtung innehaben, durch nachhaltiges und innovatives Handeln überzeugen, ganzheitlich und ergebnisorientiert denken sowie Vorbild für andere Manager und Einrichtungen sind. In der aktuellen Position sind sie mindestens fünf Jahre tätig und engagieren sich für die Profession Pflege und den Pflege-Nachwuchs. "Nur mit den entsprechenden Leitbildern auf allen Führungsebenen kann die Profession Pflege den erforderlichen Wandel erfolgreich vollziehen. Ich freue mich daher sehr, dass wir den Pflegemanagement-Award beim Kongress Pflege 2017 erstmalig in zwei Kategorien verleihen können“, so Peter Bechtel, Vorstandsvorsitzender des Bundesverbands Pflegemanagement.

www.bv-pflegemanagement.de/award.htm

\section{Deutsche Sektion von EuropaColon gegründet}

— Nur etwa jeder Fünfte nimmt in Deutschland die ihm zustehenden DarmkrebsVorsorgeuntersuchungen in Anspruch. Die neu gegründete EuropaColon Deutschland e.V. will Anlaufstelle für Darmkrebspatienten werden und zu einer risikoadaptierten Vorsorge und optimalen Behandlung beitragen. In Deutschland erkranken jährlich über 62.000 Menschen neu an Darmkrebs, der für mehr als 25.000 einen tödlichen Verlauf nimmt. Auch ist ein deutlicher Trend in Richtung jüngeres Erkrankungsalter zu beobachten, erklärte Prof. Dr. Gabriele Möslein, Wuppertal, Vorsitzende des Wissenschaftlichen Beirats von EuropaColon Deutschland e.V. Bei etwa 75\% tritt Darmkrebs sporadisch auf, 15-20\% sind familiär vorbelastet. Zu fordern sei daher eine risikostratifizierte Vorsorge. Der jetzt gegründete EuropaColon Deutschland e. V. versteht sich als Lobby für alle Darmkrebspatienten. Zu den Zielen des gemeinnützigen Vereins gehören Verbesserungen in der Vorsorge und Therapie, mehr Lebensqualität für die Erkrankten und Senkung der Mortalitätsrate, Beratungsangebote und Aufklärungsund Überzeugungsarbeit gegenüber gesundheitspolitischen Entscheidungsträgern. EuropaColon Deutschland fordert die Einführung eines leistungsfähigen, organisierten Screeningprogramms und eine systematische Früherkennung von DarmkrebsVorstufen.

Pressegespräch "Gründung von EuropaColon Deutschland e. V.", Berlin; unterstützt von der SIRTEX Medical Europe GmbH, Bonn
Alles richtig?

Die Antworten der PflegeKollegs lauten:

Krätze, Läuse \& Co.: $1 c, 2 a, 3 c, 4 b, 5 c, 6 a, 7 c, 8 b, 9 a, 10 b$

Mobil im Alter:

$1 a, 2 a, 3 c, 4 c, 5 a, 6 b, 7 c, 8 b, 9 c, 10 a$

Männersache:

1c, 2b, 3b, 4c, 5a, 6b, 7a, 8b, 9a, 10c

Organtransplantation: $1 c, 2 a, 3 c, 4 b, 5 a, 6 c, 7 a, 8 b, 9 a, 10 b$

Vorschau! Die PflegeKollegs im Monat Oktober:

Schmerz und Psyche

Chronisch - Pathologisch - Somatisch

Arzneimittelsicherheit Planung - Wirkung - Vergiftung 